\title{
MRI FINDINGS IN CEREBRAL PALSY PATIENTS- A STUDY OF 97 PATIENTS IN A TERTIARY CARE CENTRE IN SOUTH INDIA
}

\author{
Velusamy $S^{1}$, Krishnakumar $B^{2}$, Mohanakkannan $S^{3}$, Malcolm Jeyaraj4, Sakthi Velayutham ${ }^{5}$, Sowmini P. $R^{6}$, Arunan $S^{7}$ \\ ${ }_{1}^{1}$ HOD, Department of Paediatric Neurology, Stanley Medical College, Chennai, Tamilnadu, India. \\ ${ }^{2}$ Assistant Professor, Department of Paediatric Neurology, Stanley Medical College, Chennai, Tamilnadu, India. \\ ${ }_{3}^{3}$ Postgraduate Student, Department of Neurology, Stanley Medical College, Chennai, Tamilnadu, India. \\ ${ }^{4}$ Assistant Professor, Department of Neurology, Stanley Medical College, Chennai, Tamilnadu, India. \\ ${ }_{5}^{5}$ Assistant Professor, Department of Neurology, Stanley Medical College, Chennai, Tamilnadu, India. \\ ${ }^{6}$ Assistant Professor, Department of Neurology, Stanley Medical College, Chennai, Tamilnadu, India. \\ ${ }^{7}$ HOD, Department of Neurology, Stanley Medical College, Chennai, Tamilnadu, India.
}

\section{BACKGROUND}

Cerebral palsy is a common developmental disability usually attributed to birth asphyxia. But recent studies show that it may not always be true, and the aetiology of cerebral palsy has become a matter of debate.

The objectives of the present study are to determine the spectrum of MRI abnormalities in cerebral palsy and to increase our knowledge on cerebral palsy.

\section{MATERIALS AND METHODS}

A group of 97 children with the clinical diagnosis of cerebral palsy ( 52 boys and 45 girls), aged 3 months to 10 years (mean age 4.2 years) were studied prospectively. MRI was done using a 1.5 Tesla scanner. MRI was done between January and July 2017. MR images were reviewed by a radiologist for any abnormalities.

\section{RESULTS}

Periventricular leukomalacia was noted in $40 \%$ of patients. Malformations of cortical development was noted in $5.1 \%$ of patients. Corpus callosum abnormality was noted in $43.3 \%$ of patients. Diffuse cerebral and cerebellar atrophy was noted in $53.3 \%$ of patients. Arnold-Chiari and Dandy-Walker malformation was noted in $4.1 \%$ of patients. Thalamus and globus pallidus lesion was noted in $11.1 \%$ of patients. Perinatal asphyxia changes were noted in $89.7 \%$ of patients.

\section{CONCLUSION}

MRI is a useful tool in assessing the cause of insult as well as timing of insult in a cerebral palsy patient. MRI changes that are characteristic of cerebral palsy help to arrive at the diagnosis putting an end to a huge battery of tests.

\section{KEY WORDS}

MRI Findings in Cerebral Palsy, Malformations of Cortical Development, Periventricular Leukomalacia, Hypoxic Ischaemic Changes in Infants.

HOW TO CITE THIS ARTICLE: Velusamy S, Krishnakumar B, Mohanakkannan S, et al. MRI findings in cerebral palsy patients- a study of 97 patients in a tertiary care centre in South India. J. Evolution Med. Dent. Sci. 2018;7(31):3481-3484, DOI: $10.14260 /$ jemds/2018/784

\section{BACKGROUND}

Cerebral palsy is a common developmental disability that was first described by Dr. William Little in 1850 s. ${ }^{1}$ It had been widely accepted till recently that most cases of cerebral palsy were due to birth asphyxia. But recent research shows that this may not be true. One study by Nelson et al showed that cerebral palsy attributable to birth asphyxia was less than $20 \% .^{2}$ Another study by Blair et al showed that only $8 \%$ of all cerebral palsy cases were due to birth asphyxia. ${ }^{3}$ The presently accepted causes of cerebral palsy are congenital, genetic, inflammatory, infectious, anoxic and traumatic. Two important risk factors seem to be prematurity and low birth

'Financial or Other Competing Interest': None.

Submission 12-06-2018, Peer Review 17-07-2018,

Acceptance 23-07-2018, Published 30-07-2018.

Corresponding Author:

Dr. Krishnakumar B,

Assistant Professor,

Department of Paediatric Neurology,

Stanley Medical College, Chennai,

Tamilnadu, India

E-mail: kichazmd@gmail.com

DOI: $10.14260 /$ jemds $/ 2018 / 784$

\section{(c) (i) $\$$}

weight. Cerebral palsy was observed in $10-18 \%$ of babies with 500 - 999 grams birth weight. 4 The cause of Cerebral palsy is unknown in $41-58 \%$ of full-term children.5,6 The contribution of perinatal and neonatal factors is higher in pre-term infants than in full-term infants. Magnetic Resonance Imaging (MRI) can provide information about the nature of brain lesions and information about the time of occurrence of brain lesions. It is imperative to determine how much an MRI would assist in understanding the aetiology of Cerebral palsy, because it is an expensive procedure and a general anaesthetic may be required in young children to sedate them for the procedure. There have been numerous MRI studies in patients with Cerebral palsy. Truwit et al studied 40 cases and found that Cerebral palsy in 29 term infants was usually the result of factors related to prenatal period and less commonly it was related to the perinatal period. ${ }^{7}$ Periventricular Leukomalacia (PVL) was seen in $53 \%$ of term children without clinical evidence of perinatal or neonatal cause and it was thus decided to be prenatal in origin. ${ }^{8}$ Many questions thus continue to remain regarding the aetiology of Cerebral palsy. The objective of this study was to review cases of Cerebral palsy that had MRI over a defined period at a tertiary care hospital in South India, to 
determine the spectrum of MRI abnormalities in cerebral palsy and to help us improve our knowledge of cerebral palsy.

\section{MATERIALS AND METHODS}

A group of 97 children with the clinical diagnosis of cerebral palsy ( 52 boys and 45 girls), aged 3 months to 10 years (Mean age 4.2 years) were studied prospectively. All patients hailed from Chennai. The clinical presentation was developmental delay in $68 \%$ of patients, seizures in $24 \%$ of patients and failure to thrive in $6 \%$ of patients. Perinatal history of hypoxic injury was noted in 66\% (64/97) of patients. Mild mental retardation was noted in $24 \%$ of patients and moderate mental retardation in $52 \%$ of patients. Their MRI images were done between January 2017 and July 2017. Clinical details of the patients were also taken. The MRI scans were performed on 1.5 Tesla Scanner. Routinely, the sequences obtained were T1 sagittal, T2 axial, fluid attenuation inversion recovery axial images along with diffusion weighted images and apparent diffusion coefficient.
Magnetic resonance imaging scans were reviewed by one radiologist who was aware of the diagnosis of Cerebral palsy. In each patient, the images were assessed for volume and signal within the white and cortical gray matter, the basal ganglia, thalami and cerebellum. The degree of myelination and ventricular size were assessed routinely as part of examination. Any other abnormalities such as cysts or migrational abnormalities were also noted. Periventricular leukomalacia was diagnosed when the periventricular white matter demonstrated increased signal intensity on FLAIR/ T2-weighted sequences in conjunction with reduced white matter volume and associated lateral ventricular enlargement.

\section{RESULTS}

Out of 97 children with cerebral palsy, 89 (91.7\%) had abnormal neuroimaging findings.

\begin{tabular}{|c|c|c|}
\hline Sl. No. & Lesion in MRI & $\begin{array}{c}\text { No. of Patients } \\
\text { (Percentage) }\end{array}$ \\
\hline 1) & Periventricular leukomalacia and hypomyelination & $39(40 \%)$ \\
\hline 2 ) & Malformation of cortical development & $5(5.1 \%)$ \\
\hline 3$)$ & Corpus callosum abnormality & $42(43.3 \%)$ \\
\hline 4$)$ & Diffuse cerebral atrophy, cerebellar atrophy and gliosis & $53(54.6 \%)$ \\
\hline 5$)$ & Arnold-Chiari and Dandy-Walker syndrome & $4(4.1 \%)$ \\
\hline 6$)$ & Thalamus and Globus Pallidus lesion & $11(11.1 \%)$ \\
\hline 7$)$ & Vascular abnormality & $1(1.03 \%)$ \\
\hline 8$)$ & Perinatal asphyxia changes & $87(89.7 \%)$ \\
\hline
\end{tabular}

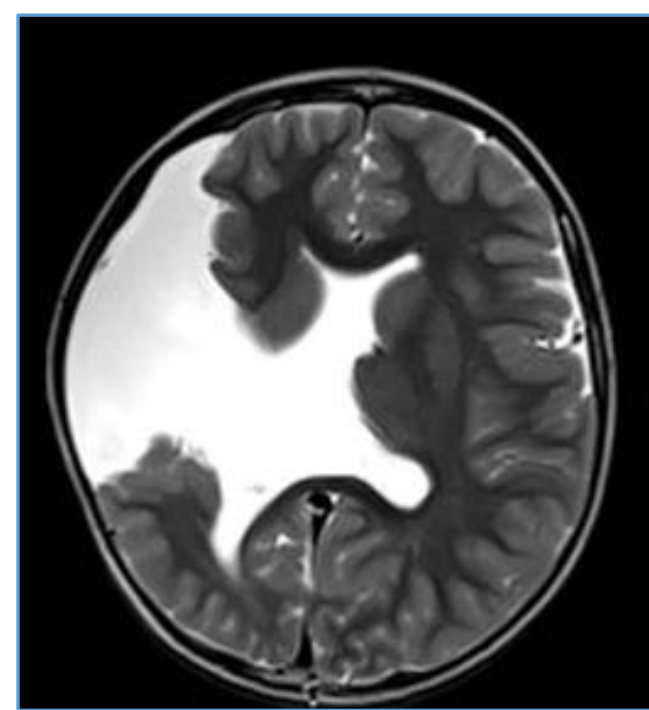

Figure 1. MRI Brain showing Schizencephaly in Right Hemisphere

Lissencephaly, which is a rare, gene-linked brain malformation characterised by the absence of normal convolutions in the cerebral cortex was noted in 1 patient. Lissencephaly is a neuronal migration anomaly where the insult occurs between 12th to 15th week of gestational age. In a study by Yin et al, lissencephaly was seen in 2 of 42 patients. Hemimegalencephaly, which is a rare neurological condition in which one-half of the brain or one side of the brain is abnormally larger than the other was noted in one patient. Heterotopia, which is a malformation of cortical development where cortical cells (grey matter) are present in inappropriate locations in the brain due to interruption in their migration to their correct location in the cerebral cortex was noted in 1 patient. Such cortical malformation are rare entities in cerebral palsy and may represent a unique finding. The finding of a hypoplastic corpus callosum is highly associated with cerebral dysgenesis as a sign of cerebral palsy. ${ }^{9}$ In our series, we found similar hypoplastic corpus callosum in 42 patients ( $43.3 \%$ of the patients). The corpus callosum is formed between the 12th and 15th week of pregnancy and thus defects in development of corpus callosum indicates that there has been insults in second trimester in cerebral palsy.10,11 Callosal thinning also could be due to white matter volume loss.

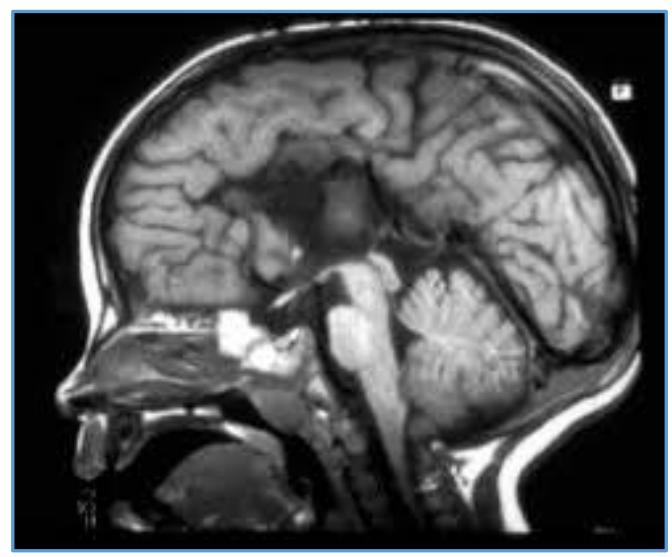

Figure 2. MRI Brain showing Absence of Corpus Callosum 
Cerebral atrophy, which is well known to be associated with cerebral palsy was noted in $53 \%$ of patients. One child had evidence for cortical infarction that is less common in a premature than in a term infant. 12 The lesion is usually found in full-term babies born after an uneventful antenatal period and a routine delivery. ${ }^{13}$ Mostly, the infarct is in distribution of the middle cerebral artery. It has been proposed that such infarcts may occur either prior to delivery or within early days of life. The key imaging features of Dandy-Walker malformations are- (a) Hypoplasia of the cerebellar vermis (whose inferior portion is the one that is typically affected), that is elevated and upwardly rotated and (b) Dilatation of the cystic-appearing fourth ventricle that may fill the whole of posterior fossa. The Arnold-Chiari malformation is a congenital anomaly, in which the cerebellar tonsils and the lower brain-stem herniate into the cervical canal. DandyWalker and Arnold-Chiari malformation were noted in 4 patients. An admixture of lesions of thalamus, putamen and peri-Rolandic area is seen in magnetic resonance imaging as a type of lesion due to HIE. The VL nucleus of thalamus, the posterior part of putamen along with pre- and post-central gyri correlate with progression of myelination and also with the metabolic activity in the brain during the time of injury. Lesions at these sites are usually thought to be caused by metabolic failure at the time of profound neonatal asphyxia. Such thalamic and globus pallidus lesions were noted in 11 patients. In a study by Krageloh Mann et al ${ }^{14}$ on 17 patients with bilateral thalamus and globus pallidus lesion, it was shown that most of these children suffered from dyskinetic cerebral palsy and some children had spastic Cerebral palsy. The severity of lesion correlated with the clinical manifestation with the more severe forms manifesting as spastic Cerebral palsy and milder forms manifesting as dyskinetic cerebral palsy.

\section{DISCUSSION}

In the 97 children who were studied, 89 children had abnormal neuroimaging findings. This is very much comparable to yield a positive MRI in other series.8,15-17 Thus, it can be safely said that a radiological diagnosis is now possible in a majority of cases of cerebral palsy. However, this radiological diagnosis may not always correlate and provide reasoning as to why the brain lesion had occurred. MRI might also provide important clue regarding the timing of the brain lesion, which can be useful not only for Cerebral palsy patients but also for any research efforts in future. Periventricular leukomalacia occurs at the age group between 28 and 34 weeks of gestation ${ }^{15}$ and it is due to an ischaemic process along the watershed zone in the periventricular white matter. The MRI features of Periventricular leukomalacia are a decrease in quantity of periventricular white matter, periventricular gliosis along with ventriculomegaly and irregular outline of the lateral ventricles. ${ }^{18,19}$ After around 34 weeks of gestation, subcortical and cortical areas are most vulnerable for hypoxic ischaemic insult 8,15 and the lesions due to it are subcortical leukomalacia and gliosis.20,21 In our study, periventricular leukomalacia was noted in $40 \%$ of the patients. This indicates that a substantial percentage of cerebral palsy patients can be attributable to hypoxic ischaemic lesions. 5 of our patients had malformation of cortical development indicating that there has been insults during second trimester of pregnancy.
The Pachygyria in which there are fewer gyri and they are abnormally broad and flat was noted in one patient. There are two basic variations in polymicrogyria, layered and nonlayered and they have differing times at onset. The nonlayered type of polymicrogyria is a disorder of neuronal migration and its time of onset appears to be usually around the fourth-to-fifth month of gestation. The layered variant of polymicrogyria are those cases that have evidence of laminar neuronal necrosis in the cortex at the end of neuronal migration, that is during the 20 and 30 weeks of gestation. ${ }^{22,23}$ Schizencephaly, which is a rare congenital (present from birth) brain malformation, in which abnormal slits or clefts form in the cerebral hemispheres of the brain was noted in 1 patient.

\section{CONCLUSION}

This article aims at giving a review of the various MRI findings that are noted in a cerebral palsy patient. Also, the 5 patients who were noted to have malformation of cortical development, represents a novel finding. We would like to conclude that MRI is a useful tool in assessing the cause of insult as well as the timing of insult in a cerebral palsy patient. MRI changes that are characteristic of cerebral palsy help to arrive at the aetiology of disease process putting an end to a huge battery of tests. Characterisation of certain surgically removable malformations of cortical development can help to decrease seizures in such children.

\section{REFERENCES}

[1] Dunn PM. Dr William Little (1810-1894) of London and cerebral palsy. Arch Dis Child 1995;72:F209-10.

[2] Nelson KB. What proportion of cerebral palsy is related to birth asphyxia? J Pediatr 1988;112(4):5724.

[3] Blair E, Stanley FJ. Intrapartum asphyxia: a rare cause of cerebral palsy. J Pediatr 1988;112(4):515-9.

[4] Msall ME. Developmental vulnerability and resilience in extremely preterm infants. JAMA 2004;292(19):2399-401.

[5] Krageloh-Mann I, Hagberg G, Meisner C, et al. Bilateral spastic cerebral palsy--a collaborative study between Southwest Germany and Western Sweden. III: Aetiology. Dev Med Child Neurol 1995;37(3):191-203.

[6] Hagberg B, Hagberg G. The origins of cerebral palsy. In: David TJ, ed. Recent advances in paediatrics. London: Churchill Livingstone 1993:67-83.

[7] Truwit CL, Barkovich AJ, Koch TK, et al. Cerebral palsy: MR findings in 40 patients. AJNR 1992;13(1):67-78.

[8] Krageloh-Mann I, Petersen D, Hagberg G, et al. Bilateral spastic cerebral palsy--MRI pathology and origin. Analysis from a representative series of 56 cases. Dev Med Child Neurol 1995;37(5):379-97.

[9] Sheth RD, Schaefer GB, Keller GM, et al. Size of the corpus callosum in cerebral palsy. J Neuroimaging 1996;6(3):180-3.

[10] Raybaud C. Destructive lesions of the brain. Neuroradiology 1983;25(4):265-91.

[11] Williams RS. Cerebral malformations arising in the first half of gestation. In: Evrard P, Minkowski A, eds. Developmental neurobiology. Vol. 12. New York: Vevey/Raven Press 1989:11-20. 
[12] Well ML, Levin M. Perinatal asphyxia and trauma. In: Menkes JH, eds. Textbook of child neurology. $5^{\text {th }}$ edn. Baltimore: Williams \& Wilkins 1995:325-78.

[13] Roach ES, Riela AR. Syndromes of vascular dysfunction. In: Pediatric cerebrovascular disorders. New York: Futura Publishing Company 1995:35-50.

[14] Krägeloh-Mann I, Helber A, Mader I, et al. Bilateral lesions of thalamus and basal ganglia: origin and outcome. Dev Med Child Neurol 2002;44(7):477-84.

[15] Steinlin M, Good M, Martin E, et al. Congenital hemiplegia: morphology of cerebral lesions and pathogenetic aspects from MRI. Neuropediatrics 1993;24(4):224-9.

[16] Candy EJ, Hoon AH, Capute AJ, et al. MRI in motor delay: important adjunct to classification of cerebral palsy. Pediatr Neurol 1993;9(6):421-9.

[17] Sugimoto T, Woo M, Nishida N, et al. When do brain abnormalities in cerebral palsy occur? An MRI study. Dev Med Child Neurol 1995;37(4):285-92.

[18] Yokochi K, Aiba K, Horie M, et al. Magnetic resonance imaging in children with spastic diplegia: correlation with the severity of their motor and mental abnormality. Dev Med Child Neurol 1991;33(1):18-25.
[19] Baker LL, Stevenson DK, Enzmann DR. End-stage periventricular leukomalacia: MR evaluation. Radiology 1988;168(3):809-15.

[20] Okumura A, Hayakawa F, Kato T, et al. MRI findings in patients with spastic cerebral palsy. I: correlation with gestational age at birth. Dev Med Child Neurol 1997;39(6):363-8.

[21] Barkovich AJ, Truwit CL. Brain damage from perinatal asphyxia: correlation of MR findings with gestational age. AJNR 1990;11(6):1087-96.

[22] Volpe JJ. Neuronal proliferation, migration, organization, and myelination. In: Neurology of the newborn. $3^{\text {rd }}$ edn. Philadelphia: WB Saunders Company 1995:43-94.

[23] Evrard P, Kadhim HJ, de Saint-Georges P, et al. Abnormal development and destructive processes of the human brain during the second half of gestation. In: Evrard P, Minkowski A, eds. Developmental neurobiology. Vol. 12. New York: Vevey/Raven Press 1989:21-41. 